\title{
Design of Ultrasonic Wind Speed Sensor for Mine
}

\author{
Yun Lei ${ }^{1,2, a}$ \\ ${ }^{1}$ Shenyang Research Institute, China Coal Technology \& Engineering Group Corp, Fushun113122, \\ China \\ ${ }^{2}$ School of Geoscience and Technology, Southwest Petroleum University, Chengdu 610500, China \\ a46960882@qq.com
}

Keywords: Mine; Wind speed sensor; Ultrasonic wave; Circuit

\begin{abstract}
The sensor used for mine was the monitoring system of coal mine. It was used to monitor the coal mine environment parameters and process parameters, and transmit various physical quantities into electrical signals. In this paper, a new type of ultrasonic wind speed sensor was designed, which had the advantages of high reliability, high adaptability and so on. Infrared remote control was used to avoid the interference flow field. In addition, a new type of single chip microcomputer and high integration digital circuit were adopted, and the circuit was simple in structure and reliable in performance.
\end{abstract}

\section{Introduction}

With the development of sensor technology, electronic, computer and information transmission technology and their application in coal mine, monitoring system, which was composed of a single parameter in early development period, has developed towards a single monitoring system with several parameters[1], in order to meet the needs of mechanized mining. These systems were based on multiple parameters monitoring in a certain aspect[2], and this includes monitoring system for rail transportation environmental safety monitoring system, monitoring system, enhancement of transportation belt transportation monitoring system, power supply system, drainage system, mine pressure monitoring system, fire control system, flood monitoring system, coal and gas outburst monitoring system, large health monitoring control system for electronic mechanical equipment, etc..

The role of mine wind speed sensor in coal mining industry is great[3]. The magnitude of wind speed directly affects the life safety of miners. Because wind speed is too small, and the harmful gas can not be diluted in time, explosion may occur. When the wind speed is too large, dust explosion may happen. Therefore, wind speed sensor is very important in coal mining.

\section{Working Principle of Ultrasonic Wind Speed Sensor}

Ultrasonic wind speed sensor is based on the principle of Kaman vortex to achieve the measurement of wind speed. Sensor output $1 \sim 5 m A D C$ analog signal, which corresponds to wind speed with $0 \sim 15 \mathrm{~m} / \mathrm{s}$. In addition, it has function of local digital display and direct reading wind speed value. It can be used to measure the wind speed in coal mine when it is used with KJ1 mine environment monitoring system. Its measuring range is $0.4 \sim 15 \mathrm{~m} / \mathrm{s}$.

\section{Hardware Structure}

The wind speed sensor is mainly composed of power supply circuit, transmitting circuit, receiving circuit, shaping circuit, frequency conversion, and direct display. Ultrasonic vortex wind speed sensor is based on the principle of Kaman vortex to achieve the measurement of wind speed. Sensor output $1 \sim 5 \mathrm{~mA} D C$ analog signal, which corresponds to wind speed with $0 \sim 15 \mathrm{~m} / \mathrm{s}$. It has display function on the spot, and can directly read the value of wind speed. Moreover, it can be used to measure the wind speed in coal mine when it is used with $\mathrm{KJ} 1$ mine environment monitoring system. Its measuring range is $0.4 \sim 15 \mathrm{~m} / \mathrm{s}$. 
Power Supply Circuit. It is composed by three terminals fixed and integrated voltage regulator W, thyratron SCR, and protection circuit which is composed of $D_{4}$ tube. Power box supply $21 \mathrm{V450mA}$ $D C$ power, and the circuit voltage output $12 \mathrm{VDC}$ voltage as the working circuit of the sensor. When $W$ is damaged due to some causes so that the output voltage is greater than $13 \mathrm{~V}, D_{4}$ zener is broken down, and thyratron SCR conduct current into ground, so as to realize the protection on the spot.

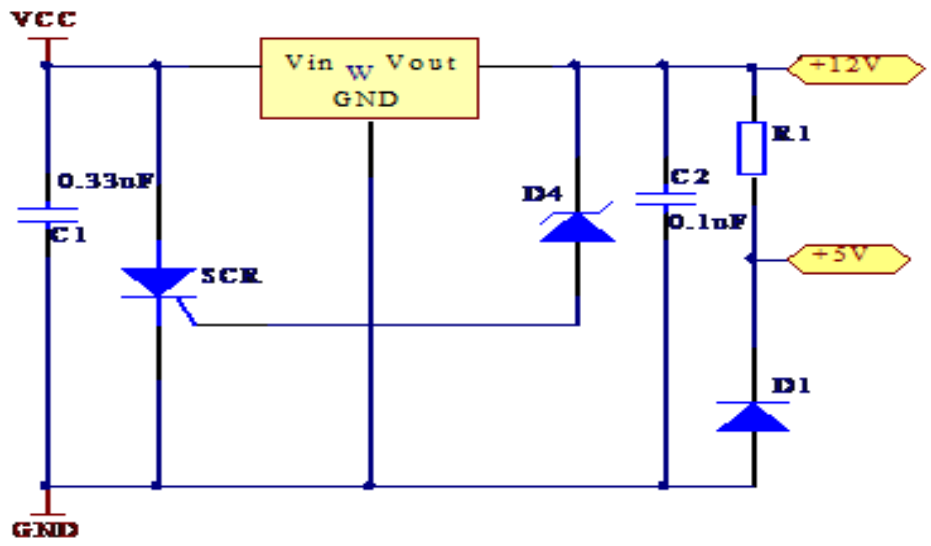

Figure 1. Finite Power circuit

Transmission Circuit. The circuit consists of an inductor three points oscillator and a class $B$ push-pull power amplifier. The oscillator generates a continuous sine wave of $141.5 \mathrm{KHz}$, which is applied to the transmitting transducer $F$ by the power input of the transformer. The transmitting voltage is about $11 \mathrm{~V}$, and the power is about $200 \mathrm{~mW}$.

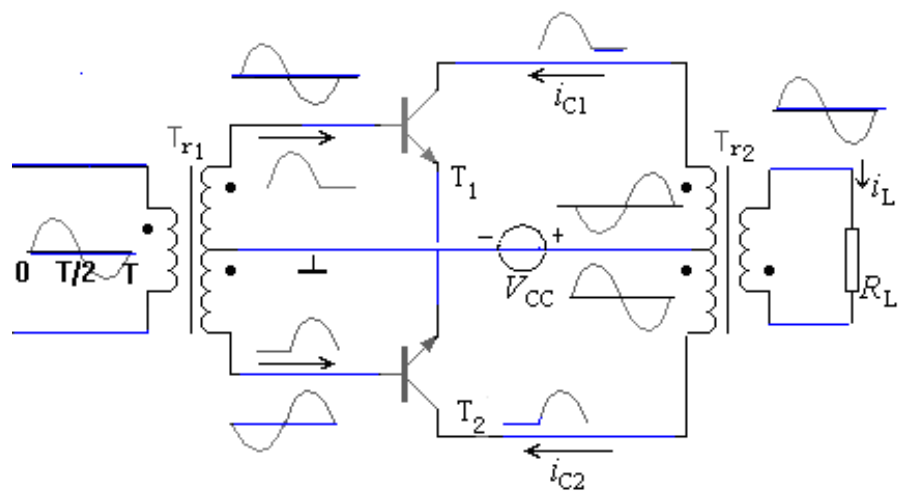

Figure 2. Finite Class $B$ push-pull power amplifier

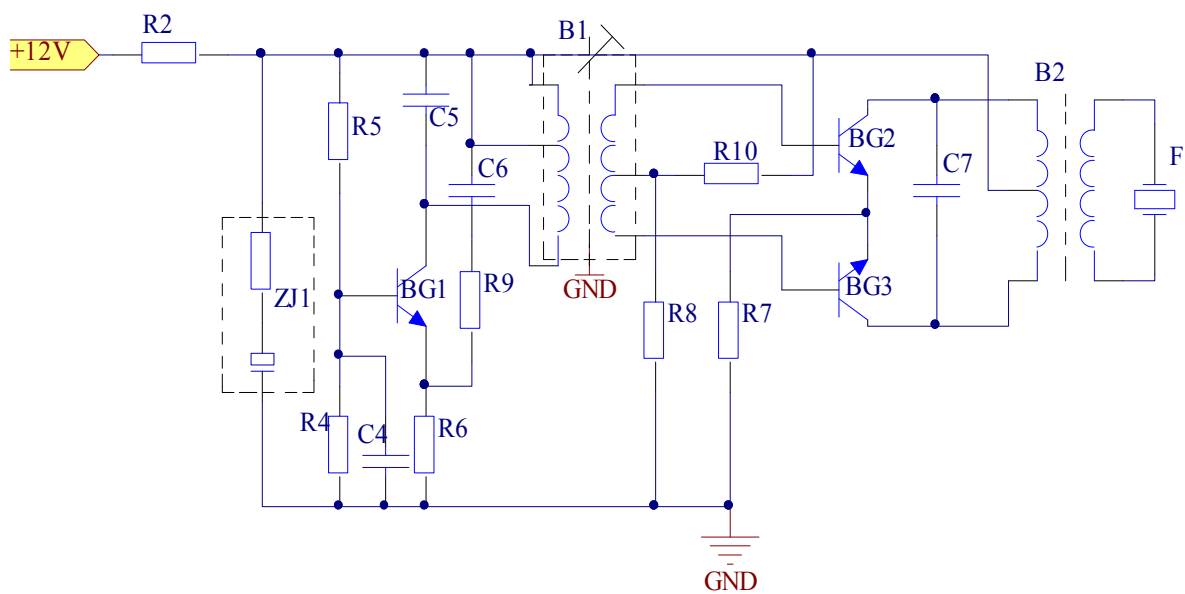

Figure 3. Finite Transmission circuit 
Receiving Circuit. Receiving circuit includes intermediate frequency amplifier, detector, and low frequency amplifier. Ultrasonic emission emitted by the transducer is received by the receiving transducer after air attenuation. Energy conversion loss is large, and the signal receiving transducer output is weak, generally only a few millivolts. In order to meet the needs of large signal detector, intermediate frequency amplifier is used to receive transducer output signal amplification. Intermediate frequency amplifier consists of two LC frequency amplifiers. Concretely, amplifier center frequency is $141.5 \mathrm{KHz}$, bandwidth is $3 \mathrm{KHz}$, voltage amplification is 600 800 times, output voltage is $1 \mathrm{~V}$, and maximum output noise voltage is not greater than $4 \mathrm{mV}$ when the input shorted.

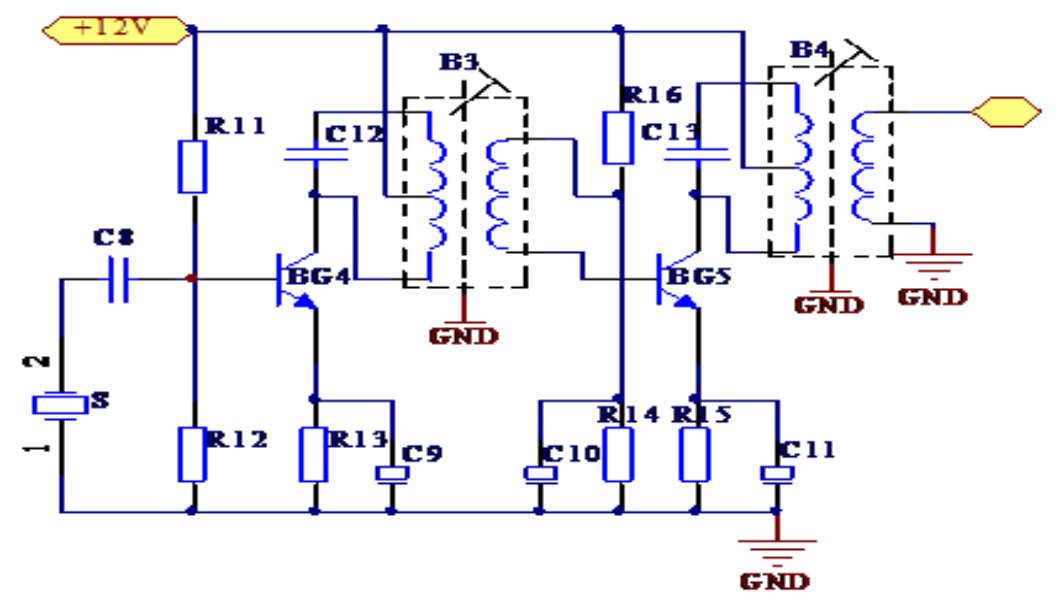

Figure 4. Finite Intermediate frequency amplifier

The detector detects the low-frequency vortex signal in the amplitude modulation signal output from the intermediate frequency amplifier to the low frequency amplifier, and the output voltage amplitude of the detector is $5 \sim 10 \mathrm{mV}$, which increases with the increase of wind speed.

Low frequency amplifier uses $8 F C 7$ single power amplifier to form a two-class amplifier. Each level is about 20 times, and the frequency range is $20 \sim 1200 \mathrm{~Hz}$. When the input end makes short circuit, the output noise voltage is not greater than $1 \mathrm{mV}$.

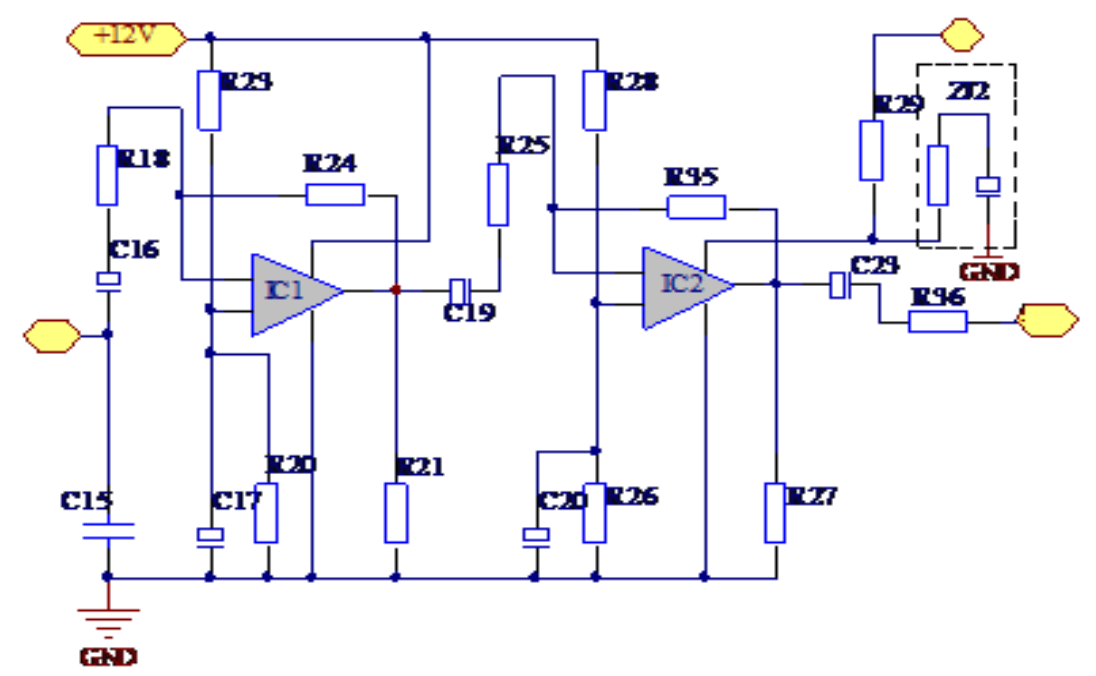

Figure 5. Finite Low frequency amplifier

Shaping Circuit. It is composed of two silicon transistors named $B G_{6}$ and $B G_{7}$, the approximate sine wave signal is transformed into low frequency rectangular wave amplifier output to complete waveform transform. One way is transmit to circuit on the spot, and another way is transmit to frequency-current conversion circuit. 


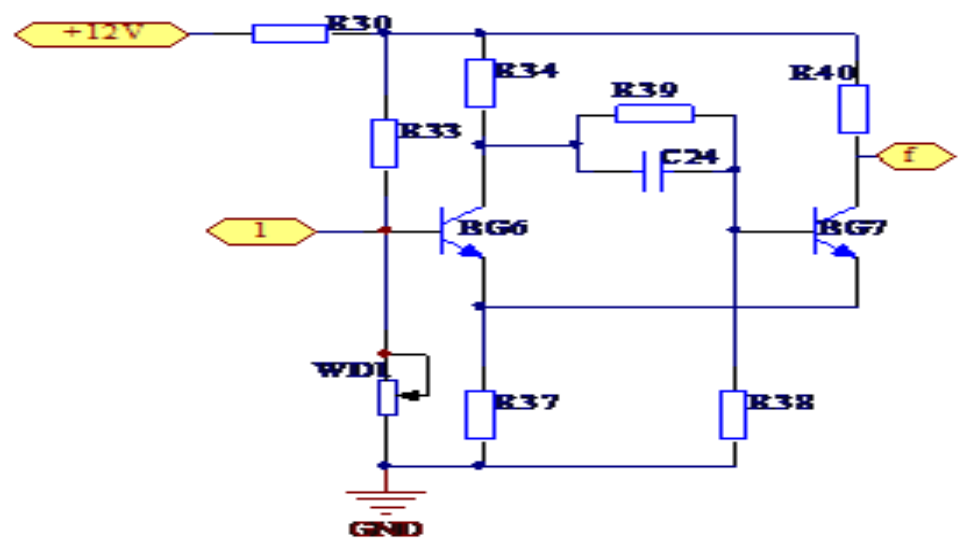

Figure 6. Finite Shaping circuit

Frequency-current Conversion Circuit. Frequency-current conversion circuit consists of $C M O S$ monostable trigger $I C_{4}$, single supply operational amplifier $I C_{5}$, field effect transistor $B G_{8}$ and silicon transistor $B G_{9}$. Monostable trigger $I C_{4}$ output pulse $T M$ is determined by $R_{42}$ and $C_{26}$, rectangular wave signal input by Schmidt is output rectangular pulse with constant pulse width after reshaping. $0 \sim 1 V D C$ voltage signal is output after pressure filter by $R_{43}, W D_{2}, R_{41}, R_{46}$, and $C_{27}$. The output voltage signal can be adjusted in a small range by $W D_{2} . I C_{5}, B G_{8}$ and $B G_{9}$ constitute a constant current circuit. $W D$ is analog load resistance, $W D_{5}$ is a non inverting amplifier sampling resistor, and $I C_{5}$ working state. The constant current transform circuit $0 \sim 1 \mathrm{VDC}$ voltage signal into $1 \sim 5 \mathrm{~mA} D C$ current signal, and the line is transported to the mine monitoring system, power supply box, thus completing the frequency current conversion.

Digital Display Circuit. CMOS can timely control $I C_{6}$, which is composed of decimal digital storage decoder $I C_{7}, I C_{8}, I C_{9}$ and digital tube, etc.. The timely controller $I C_{6}$ consists crystal oscillator $S Z$ and $R_{56}, C_{30}, C_{31}$, producing frequency of $32768 \mathrm{~Hz} .12$ feet of $I C_{6}$ output $32 \mathrm{~Hz}$, square wave signal with $50 \%$ duty cycle is the driving signal of digital tube.

\section{Summary}

In this paper, a new type of ultrasonic wind speed sensor was designed, which has the advantages of high reliability, high adaptability and so on. Infrared remote control was used to avoid the interference flow field. In addition, a new type of single chip microcomputer and high integration digital circuit were adopted. The circuit was simple in structure and reliable in performance, and the shell was made of stainless steel material, which enhanced the shock resistance and corrosion resistance of the sensor. Under the continuous testing, has the following advantages: No moving parts, no mechanical wear, stable performance, long service life. The output itself was a linear relationship between the pulse frequency signal and wind speed, no zero drift and the sensor sensitivity change does not directly affect the output, high measurement precision; quick response.In addition, the output signal was not affected by fluid properties, such as temperature, humidity, pressure, composition, density, viscosity, dust etc..

\section{Acknowledgements}

This work was financially supported by the National Science and Technology Major Project (Grant No. 2016ZX05067).

\section{References}

[1] DU Wei-Lue,XP Tan, Measurement \& Control Technology, Vol. 12 (2013) No.9, p.62.

[2] XS Xin,K Zhou, Instrument Technique \& Sensor, Vol. 22 (2012) No.8, p.31.

[3] K Xie,K Wang, Energy Procedia,Vol. 34 (2011) No.12, p.837. 